\title{
Discrepancy based control of continuous fluidized bed spray granulation with internal product classification
}

\author{
Stefan Palis ${ }^{1, *}$, Achim Kienle ${ }^{1,2}$ \\ 1 Otto-von-Guericke-Universität, \\ Universitätsplatz 2, D-39106 Magdeburg, Germany \\ 2 Max-Planck-Institut für Dynamik komplexer technischer Systeme \\ Sandtorstrasse 1, D-39106 Magdeburg, Germany
}

\begin{abstract}
This paper is concerned with stabilizing open loop unstable fluidized bed spray granulation with internal product classification by means of nonlinear feedback control. The process dynamics are described by a population balance model, i.e. a nonlinear partial integrodifferential equation. Therefore a direct stabilization in a classical $L_{p}$-norm is at least very difficult. To overcome this problem a stability concept using two generalized distance measures, called discrepancies, is used. In order to show that the discrepancy based control approach presented in this contribution also guarantees stability in the sense of a $L_{p}$-norm or $L_{\infty}$-norm additional conditions are stated and verified.
\end{abstract}

\section{INTRODUCTION}

Fluidized bed spray granulation is a particulate process, where a bed of particles is fluidized, while simultaneously injecting a solid matter suspension or solution. As process air temperatures are typically high, the fluid evaporates and the remaining solid material either forms new nuclei or contributes to growth of already existing particles. In order to guarantee a minimum product particle size a product classification is required. This can be either done by external classification using sieves with corresponding recycle of the over- and undersized fraction [2] or by internal classification using an air sifter with countercurrent flow [4]. The focus in this work is on continuous fluidized bed spray granulation with internal product classification as depicted in Fig. 1. A corresponding process model has been proposed recently by Vreman et. al. [4]. In partic-

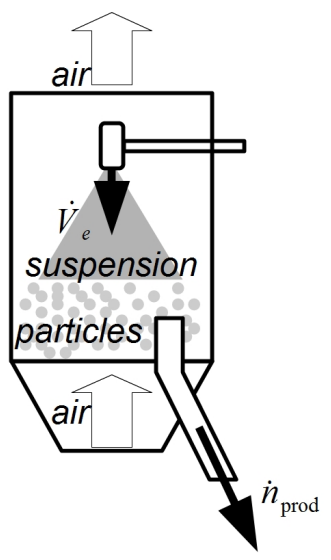

Fig. 1. Process scheme

ular, it has been shown that for certain ranges of the operating parameters regions of instability, resulting in nonlinear oscillations of the particle size distribution, exist. These oscillations give undesired time behavior of product quality. Similar patterns of behavior have been observed for other particulate processes as continuous fluidized bed spray granulation with external product classification and material recycles [2, 3] and crystallization processes [14]. So far, main emphasis was on crystallization processes. Here, several approaches for stabilizing control design have been proposed, using linear (e.g. [11]) and nonlinear (e.g. [12]) lumped models or linear (e.g. [13]) and nonlinear infinite dimensional models, i.e. applying the discrepancy based control approach presented in this contribution [9]. For fluidized bed spray granulation with external product classification a linear and nonlinear control design have been proposed recently in $[6,7]$.

In this contribution control design based on the nonlinear infinite dimensional model of the fluidized bed spray granulation with internal product classification is investigated using the concept of stability in the sense of two generalized distances, called discrepancies.

\section{CONTINUOUS FLUIDIZED BED SPRAY GRANULATION WITH CLASSIFYING PRODUCT WITHDRAWAL}

Continuous fluidized bed spray granulator with internal product classification as depicted in Fig. 1 consists of a granulation chamber, where the particle population is fluidized through an air stream and coated by injecting a suspension $\dot{V}_{e}$. The particle growth associated to the layering process has been described in [1]. In the present configuration only a certain part of the injected suspension $(1-b) \dot{V}_{e}$ contributes to the particle growth, while the rest $b \dot{V}_{e}$ completely dries before hitting existing particles in the bed.

$$
G=\frac{2(1-b) \dot{V}_{e}}{\pi \int_{0}^{\infty} L^{2} n d L}=\frac{2(1-b) \dot{V}_{e}}{\pi \mu_{2}}
$$


In the following, the moments $\mu_{i}$ of the particle size distribution $n$ are defined as follows

$$
\mu_{i}=\int_{0}^{\infty} L^{i} n d L
$$

For the generation of new particles it is assumed that nuclei with a characteristic diameter $L_{0}$ are formed due to spray drying.

$$
B=\frac{b \dot{V}_{e}}{\frac{1}{6} \pi L_{0}^{3}} \delta\left(L-L_{0}\right)
$$

The nucleation parameter $b$ determining how much of the injected suspension results in new particles is assumed to depend only on the bed height $h$, which can be obtained from

$$
h=\frac{V}{(1-\varepsilon) A},
$$

where $\varepsilon$ is the bed porosity. In the following the bed porosity $\varepsilon$ is assumed to be constant. The free distance for the spray droplets decreases with increasing bed height $h$ resulting in a decreasing nuclei formation. For a bed higher or equal to the nozzle height a constant minimal nucleation parameter $b=b_{\infty}$ is assumed. The maximum of the nucleation parameter $b=1$ is reached for a minimum bed height of 0 resulting in a pure spray drying process, i.e. $100 \%$ of the injected suspension forms new particles. For bed heights between the two extremal situations $h=0$ and $h=h_{n o z}$ the nucleation parameter $b$ is interpolated linearly.

$$
b=b_{\infty}+\max \left(0,\left(1-b_{\infty}\right) \frac{h_{n o z}-h}{h_{n o z}}\right)
$$

In order to guarantee a continuous process operation particles are continuously removed through an air sifter with countercurrent flow. Due to the particle size specific sinking velocity large particles pass the air sifter while small particles are reblown into the granulation chamber. The minimum particle size, which can pass through the air sifter is characterized by the sifting diameter $L_{1}$. The classifying product removal therefore reads

$$
\dot{n}_{\text {prod }}=K \sigma\left(L-L_{1}\right) n
$$

where $K$ is the drain. As has been shown in [4] the process can be described by a population balance model for the particle size distribution consisting of the following particle fluxes:

- $B$ particle flux due to nuclei formation,

- $\dot{n}_{\text {prod }}$ particle flux due to product removal,

and size independent particle growth associated with the particle growth rate $G$

$$
\frac{\partial n}{\partial t}=-G \frac{\partial n}{\partial L}-\dot{n}_{\text {prod }}+B
$$

For simulation the model equations are discretized applying the finite volume method (1st order upwind flux discretization) with 150 grid points. The model parameters used are given in Tab. 1.

\begin{tabular}{ll}
\hline$A$ & $5 \cdot 10^{6} \mathrm{~mm}^{2}$ \\
$h_{n o z}$ & $440 \mathrm{~mm}$ \\
$\varepsilon$ & 0.5 \\
$\dot{V}_{e, 0}$ & $1.67 \cdot 10^{5} \frac{\mathrm{mm}^{3}}{\mathrm{~s}}$ \\
$b_{\infty}$ & 0.028 \\
$L_{0}$ & $0.3 \mathrm{~mm}$ \\
$L_{1}$ & $0.7 \mathrm{~mm}$ \\
$K$ & $1.92 \cdot 10^{-4} \frac{1}{\mathrm{~s}}$ \\
\hline
\end{tabular}

Table 1. Plant parameters

For this model interesting dynamical behavior can be observed, when starting with an initial particle size distribution equal to the steady state particle size distribution for $\dot{V}_{e, 0}=16700 \frac{\mathrm{mm}^{3}}{\mathrm{~s}}$. For sufficiently high suspension injection rates and an associated bed height higher than the nozzle height, transition processes decay and the particle size distribution reaches a stable steady state as shown in Fig. 2. Decreasing the suspension injection rate below a critical value the steady state becomes unstable giving rise to nonlinear oscillations as depicted in Fig. 3. Here, the associated mechanism is as follows:

- For a bed height smaller than the nozzle height spray drying causes an increased nuclei production.

- This results in an increasing number of smaller particles and a reduced growth rate.

- When the bed height reaches the nozzle height the nuclei production becomes small and remains constant, resulting in a higher growth rate.

- However, when the peak of the particle size distribution reaches the critical air sifter radius $L_{1}$ the associated particles are removed from the granulator resulting in a decrease of the bed height below the nozzle height and hence the process repeats.

In contrast, a high suspension rate gives a constantly high nuclei production and a high growth rate. Therefore, the bed height remains bigger than the nozzle height and after a transition time the steady state particle size distribution is reached and no oscillations occur.
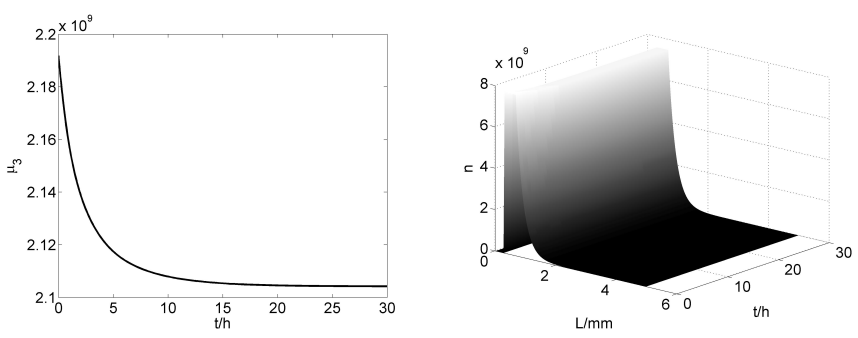

Fig. 2. Open loop Simulation in the stable region $\dot{V}_{e}=$ $0.96 \cdot \dot{V}_{e, 0}-\mu_{3}$ (left) and $n$ (right)

A detailed one-parameter bifurcation analysis (Fig. 4) confirms the previously described process time behavior and its dependence on the chosen suspension injection rate $\dot{V}_{e}$. For sufficiently high values of $\dot{V}_{e}$ the particle size distribution reaches a stable steady state. Decreasing the suspension injection rate $\dot{V}_{e}$ to a critical value and below leads to a loss of stability for the steady state solution and a stable limit cycle occurs. The stable limit cycle is associated to undesired self-sustained oscillations. 

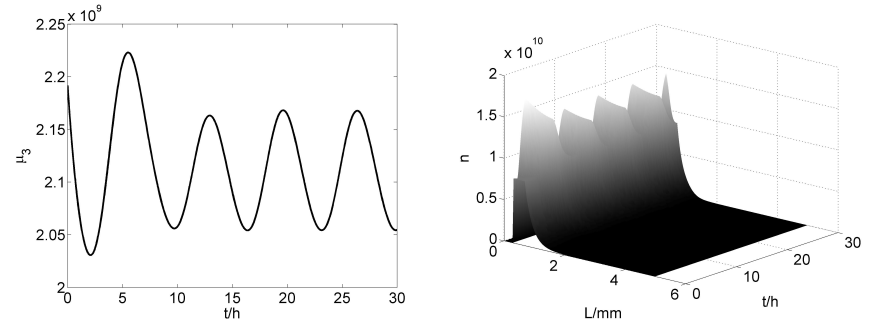

Fig. 3. Open loop Simulation in the unstable region $\dot{V}_{e}=$ $0.9 \cdot \dot{V}_{e, 0}-\mu_{3}$ (left) and $n$ (right)
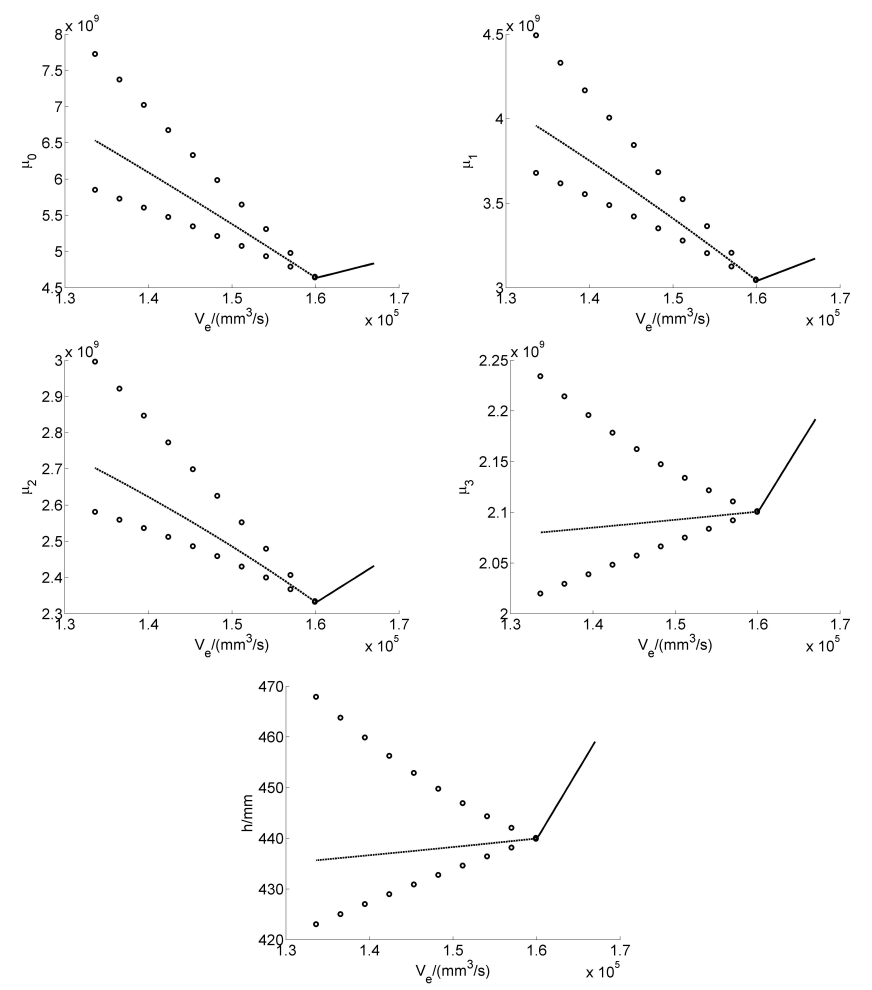

Fig. 4. One-parameter bifurcation diagrams depending on $\dot{V}_{e}$

Therefore in the following a stabilizing control will be designed. As we showed by a local controllability analysis [10] the suspension injection rate $\dot{V}_{e}$ can be used as a control input. This is in accordance to our earlier results on control of fluidized bed spray granulation with external product classification and mill cycle $[6,7,8]$, where we choose the mill grade, i.e. the principal bifurcation parameter, as control input.

\section{STABILITY WITH RESPECT TO TWO DISCREPANCIES}

Over the last decades different methods for the stabilization of systems with distributed parameters have been developed. Most of them are based on the solution of the system itself or at least the desired error system, i.e. the system in closed loop operation. In the backstepping approach [15] for example the control input is designed such that it maps the original system onto a desired stable error system. Whereas in the works of Bastin et. al. [16] stability is proven using the solution derived with the method of characteristics.
Here, the population balance is a nonlinear partial integrodifferential equation with limited control input and a lack of solution theory. Hence, in this case a transformation to a desired error system with known classical stability behavior is hardly possible. This problem is however solvable by introducing a generalized stability notion in the sense of two generalized distance measures, called discrepancies. In the following, the most important properties and facts on stability with respect to two discrepancies are stated in accordance to $[17,18,19]$. Here, the process $\varphi(., t)$ is a solution of the distributed parameter system and $\varphi_{0}=0$ an equilibrium of the system.

Definition 1. Discrepancy

A discrepancy is a real valued functional $\rho=\rho[\varphi(., t), t]$ with the following properties

(1) $\rho(\varphi, t) \geq 0$

(2) $\rho(0, t)=0$

(3) for an arbitrary process $\varphi=\varphi(., t)$ the real valued functional $\rho(\varphi(., t), t)$ is continuous with respect to $t$.

The discrepancy $\rho(\varphi(., t), t)$ is a measure of the distance between the process $\varphi(., t)$ and the equilibrium $\varphi_{0}$. Any measure of distance with the above described properties can be used. Of great importance is the fact that a discrepancy has not all properties of a metric, e.g. symmetry $d(x, y)=d(y, x)$ or triangular inequality $d(x, y) \leq$ $d(x, z)+d(z, y)$. In addition, it has not to satisfy the important property of definiteness, i.e a vanishing discrepancy $\rho(\varphi, t)=0$ does not automatically imply $\varphi=0$. Therefore, the discrepancy is an extension of the distance measures normally used in stability theory for distributed parameter systems like $L_{p}$ and $L_{\infty}$-norms.

In the context of stability with respect to two discrepancies beside the discrepancy $\rho(\varphi(., t, t))$ measuring the distance between $\varphi(., t)$ and the equilibrium $\varphi_{0}$ a second time independent discrepancy $\rho_{0}$ is used describing the distance between the initial state $\varphi(., 0)$ and the equilibrium $\varphi_{0}$. The two discrepancies $\rho$ and $\rho_{0}$ have to satisfy, that the discrepancy $\rho(\varphi(., t), t)$ is continuous at time $t=t_{0}$ with respect to $\rho_{0}$ at $\rho_{0}=0$, i.e. for every $\varepsilon>0$ and $t_{0}>0$ there exists a $\delta\left(\varepsilon, t_{0}\right)>0$, such that from $\rho_{0} \leqslant \delta\left(\varepsilon, t_{0}\right)$ follows $\rho<\varepsilon$.

Definition 2. Stability with respect to two discrepancies $\rho$ and $\rho_{0}$

The equilibrium $\varphi_{0}=0$ is stable in the sense of Lyapunov with respect to the two discrepancies $\rho$ and $\rho_{0}$ for all $t \geq t_{0}$ if for every $\varepsilon>0$ and $t_{0} \geq 0$ there exists a $\delta=\delta\left(\varepsilon, t_{0}\right)>0$, such that for every process $\varphi(., t)$ with $\rho_{0}<\delta\left(\varepsilon, t_{0}\right)$ follows $\rho<\varepsilon$ for all $t \geq t_{0}$. If in addition $\lim _{t \rightarrow \infty} \rho=0$, than the equilibrium $\varphi_{0}$ is called asymptotically stable in the sense of Lyapunov with respect to the two discrepancies $\rho$ and $\rho_{0}$.

The basis of many nonlinear control methods is the stability theory of Lyapunov (e.g. [21]). The knowledge of a control Lyapunov function for example immediately allows the design of a stabilizing control (e.g. [22]). In order to achieve a comparable situation for the stability with respect to two discrepancies a relationship between the existence of a Lyapunov functional $V$ and stability with respect of two discrepancies has to be established. For this purpose the notions of positivity and positive definiteness 
of a functional with respect to a discrepancy are introduced.

Definition 3. Positivity with respect to a discrepancy $\rho$

The functional $V=V[\varphi, t]$ is called positive with respect to the discrepancy $\rho$, if $V \geq 0$ and $V[0, t]=0$ for all $\varphi$ with $\rho(\varphi, t)<\infty$.

Definition 4. Positive definiteness with respect to a discrepancy $\rho$

The functional $V=V[\varphi, t]$ is positive definite with respect to a discrepancy $\rho$, if $V \geq 0$ and $V[0, t]=0$ for all $\varphi$ with $\rho(\varphi, t)<\infty$ und for every $\varepsilon>0$ there exists a $\delta=\delta(\varepsilon)>0$, such that $V \geq \delta(\varepsilon)$ for all $\varphi$ with $\rho[\varphi, t] \geq \varepsilon$.

The following two theorems state the conditions for a functional $V$ guaranteeing (asymptotic) stability with respect to two discrepancies.

Theorem 1. [18] The process $\varphi$ with the equilibrium $\varphi_{0}=$ 0 is stable with respect to the two discrepancies $\rho$ and $\rho_{0}$ if and only if there exists a functional $V=V[\varphi, t]$ positive definite with respect to the discrepancy $\rho$, continuous at time $t=t_{0}$ with respect to $\rho_{0}$ at $\rho_{0}=0$ and not increasing along the process $\varphi$, i.e. $\dot{V} \leq 0$.

Theorem 2. [18] The process $\varphi$ with the equilibrium $\varphi_{0}=$ 0 is asymptotically stable with respect to the two discrepancies $\rho$ and $\rho_{0}$ if and only if there exists a functional $V=V[\varphi, t]$ positive definite with respect to the discrepancy $\rho$, continuous at time $t=t_{0}$ with respect to $\rho_{0}$ at $\rho_{0}=0$ and not increasing along the process $\varphi$, i.e. $\dot{V} \leq 0$, with $\lim _{t \rightarrow \infty} V=0$.

\section{DISCREPANCY BASED CONTROL DESIGN}

In the following a stabilizing control is derived for the fluidized bed spray granulation with internal classification (7). As has been shown earlier [10] the third moment of the particle size distribution $\mu_{3}$ as the controlled variable and the suspension injection rate $\dot{V}_{e}$ as the control variable are appropriate handles in order to stabilize the process. The error therefore is

$$
e=\int_{0}^{\infty} L^{3}\left(n_{d}-n\right) d L
$$

In order to derive a stabilizing controller the above presented stability concept is applied. Here, we choose the discrepancy $\rho$ as follows

$$
\rho=\frac{1}{2}\left(\int_{0}^{\infty} L^{3}\left(n_{d}-n\right) d L\right)^{2}
$$

Obviously, the above requirements on a discrepancy are met. In order to guarantee continuity at time $t=t_{0}$ at $\rho_{0}=0$ the second discrepancy $\rho_{0}$ is chosen as follows

$$
\rho_{0}=\rho(t=0) .
$$

According to Theorem 2 existence of an appropriate functional $V$ is sufficient to guarantee asymptotic stability with respect to the two discrepancies $\rho$ and $\rho_{0}$. For this purpose the following candidate Lyapunov functional is introduced

$$
V=\frac{1}{2}\left(\int_{0}^{\infty} L^{3}\left(n_{d}-n\right) d L\right)^{2} .
$$

In order to achieve stability in the sense described above the control variable has to be chosen such that the time derivative of $V$ along the system trajectories (7) is negative definite for all times and vanishs only for $V=0$.

$$
\dot{V}\left\{\begin{array}{l}
<0 \text { for } V \neq 0 \\
=0 \text { for } V=0 .
\end{array}\right.
$$

Calculating the time derivative $\dot{V}$ yields

$$
\begin{aligned}
\dot{V}= & -e\left[\int_{0}^{\infty} L^{3}\left(-G \frac{\partial n}{\partial L}-\dot{n}_{\text {prod }}+B\right) d L\right] \\
= & -e \int_{0}^{\infty} L^{3}\left(-\frac{2(1-b)}{\pi \mu_{2}} \frac{\partial n}{\partial L}+\frac{b}{\frac{1}{6} \pi L_{0}^{3}} \delta\left(L-L_{0}\right)\right) \dot{V}_{e} d L \\
& -e \int_{0}^{\infty} L^{3} \dot{n}_{\text {prod }} d L .
\end{aligned}
$$

Using (14) the negative definiteness of the time derivative of the candidate Lyapunov functional $V$ can be guaranteed choosing the following control law

$$
\dot{V}_{e}=\frac{c e+\int_{0}^{\infty} L^{3} \dot{n}_{\text {prod }} d L}{\int_{0}^{\infty} L^{3}\left(-\frac{2(1-b)}{\pi \mu_{2}} \frac{\partial n}{\partial L}+\frac{b}{\frac{1}{6} \pi L_{0}^{3}} \delta\left(L-L_{0}\right)\right) d L},
$$

where $c$ is a positive constant $\left(c=3 \cdot 10^{-4}\right)$. The control law as depicted in Fig. 5 consists of nonlinear compensation part, which needs a measurement of the particle size distribution $n$, and of a proportional error feedback.

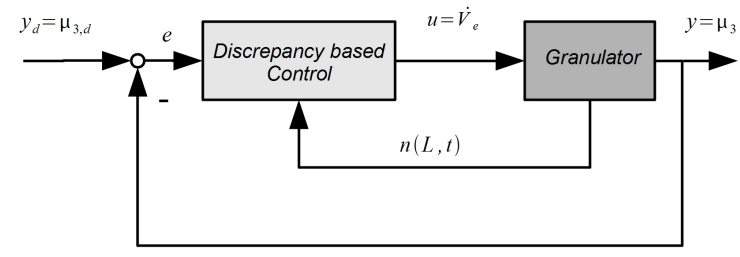

Fig. 5. Control scheme

In addition to stability, the control law (15) guarantees exponential convergence of $V$.

$$
\dot{V}=-c e^{2}=-2 c V
$$

However, it has to be mentioned that stability with respect to two discrepancies is necessary but in general not sufficient for stability with respect to a $L_{p}$-norm or pointwise convergence. In order to state conditions, when convergence in two discrepancies yields convergence of the deviation $n_{e}$ of the particle size distribution $n$ to a desired particle size distribution $n_{d}$, the following well known results from finite dimensional system theory (e.g. [20]) can be generalized to the infinite dimensional case. Consider for example the following first-order partial differential equation. 


$$
\begin{aligned}
\frac{\partial w}{\partial t} & =A(x) \frac{\partial w}{\partial x}+B(x) u \\
y & =C(x) w
\end{aligned}
$$

Taking successively time derivatives of the output $y$ yields:

$$
\begin{aligned}
y= & C(x) w, \\
\dot{y}= & C(x) A(x) \frac{\partial w}{\partial x}+\underbrace{C(x) B(x)}_{=0} u, \\
& \vdots \\
y^{(j)}= & C(x)\left(A(x) \frac{\partial}{\partial x}\right)^{j} w \\
& +C(x)\left(A(x) \frac{\partial}{\partial x}\right)^{j-1} B(x) u .
\end{aligned}
$$

In analogy with the definition of the relative degree for finite dimensional systems (e.g. [20]) the relative degree is defined as the first index $j$ for which $C(x)\left(A(x) \frac{\partial}{\partial x}\right)^{j-1} B(x)$ does not vanish, i.e. $C(x)\left(A(x) \frac{\partial}{\partial x}\right)^{j-1} B(x) \neq 0$. As a separation of the state variables is inconvenient for a distributed parameter system, the zero dynamics are defined by constraining the output to zero in closed loop operation. It is well known, that a system with a relative degree smaller than the system order is stabilized applying a linearizing control law based on the output $y$ and input $u$ if and only if the associated zero dynamics are stable. Therefore, applying the discrepancy based control law guarantees stability of the whole system if and only if the zero dynamics associated with the discrepancy $\rho$ are stable. Unfortunately, this condition is very hard to check. Thus, for the fluidized bed spray granulation with internal product classification only the conditions related to the finite dimensional linear case, i.e. the presence of righthalf plane zeros, will be checked. This gives at least a local result, guaranteeing stability in a neighborhood of a steady state. As can be seen from the pole/zero map in Fig. 6 the linear approximations around the steady states for varying $\dot{V}_{e}$ have no zeros in the right half plane. Hence, the zero dynamics are stable and stability with respect to the discrepancy $\rho$ yields pointwise convergence of the deviation $n_{e}$ of the particle size distribution $n$ to a desired particle size distribution $n_{d}$.

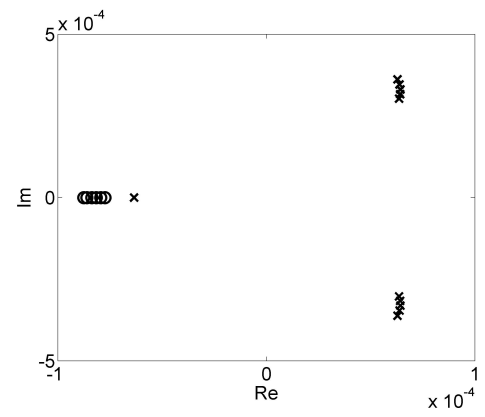

Fig. 6. Pole/zero map for varying $\dot{V}_{e}$

\subsection{Simulation scenario I}

In order to test the control law the desired third moment $\mu_{3, d}$ is shifted by a ramp from its steady state value for $\dot{V}_{e}=16700 \frac{\mathrm{mm}^{3}}{\mathrm{~s}}$ in the stable region to its steady state value for $\dot{V}_{e}=133600 \frac{\mathrm{mm}^{3}}{\mathrm{~s}}$ in the unstable region. For comparison a shift of suspension rate from $\dot{V}_{e}=$ $16700 \frac{\mathrm{mm}^{3}}{\mathrm{~s}}$ to $\dot{V}_{e}=133600 \frac{\mathrm{mm}^{3}}{\mathrm{~s}}$ in open loop operation has been calculated. As can be seen in Fig. 7 in closed loop operation the particle size distribution $n$ and all its moments $\mu_{0}, \mu_{1}, \mu_{2}, \mu_{3}$ are stabilized with reasonable control effort.
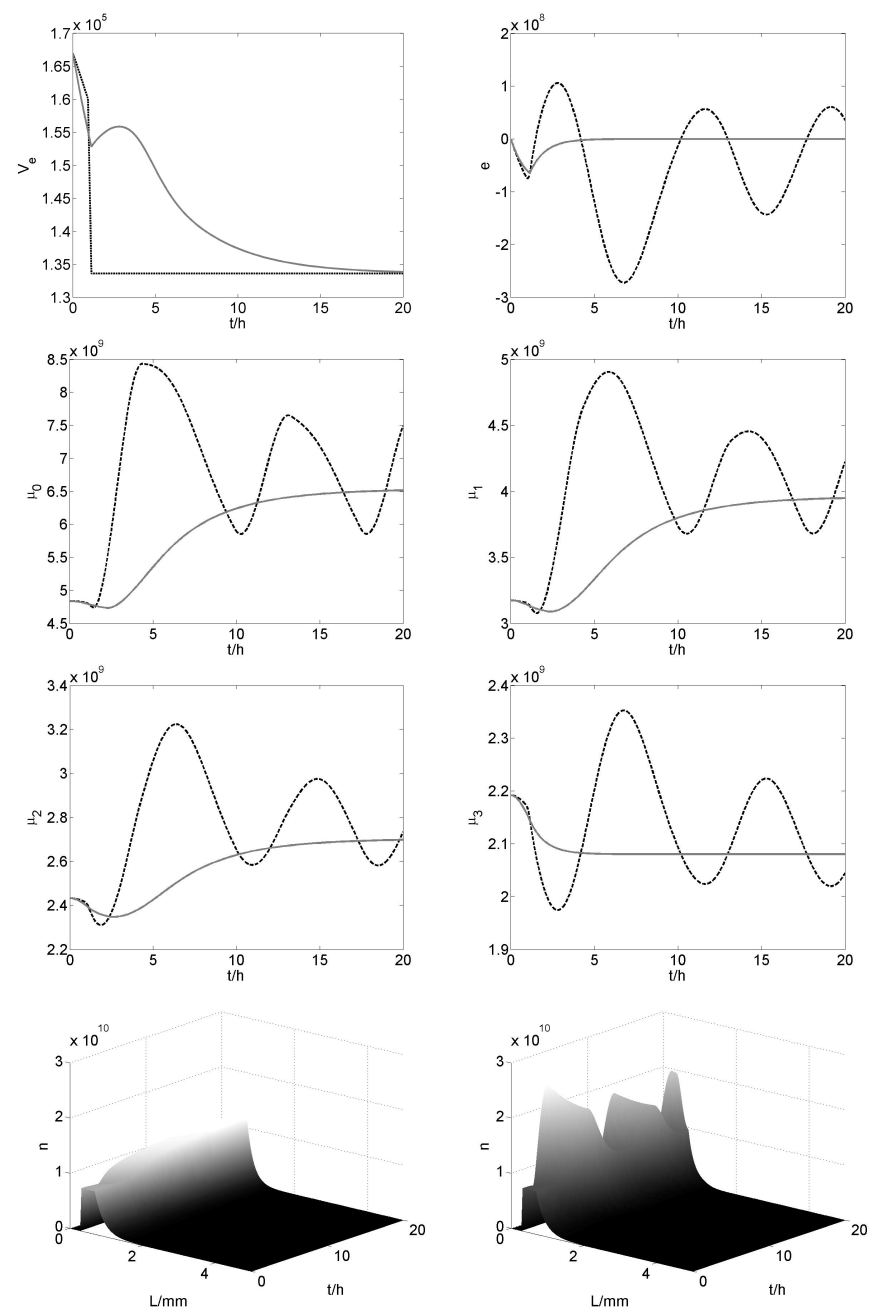

Fig. 7. Set point shift with (gray) and without control (black - -)

\subsection{Simulation scenario II}

In simulation scenario I the discrepancy based control law has been tested for its local behavior, i.e. the slow shift of the desired particle size distribution guaranteed that the process stays close to its steady state. Assuming that the aforementioned zero dynamics are stable not only in a neighborhood of the steady state the proposed discrepancy based control law is applied in order to stabilize the particle size distribution directly from the limit cycle. Therefore, the control loop is closed after $30 h$. As can be seen in Fig. 8 the proposed discrepancy based control law is able to stabilize the particle size distribution $n$ and all its moments $\mu_{0}, \mu_{1}, \mu_{2}, \mu_{3}$ with reasonable control effort starting directly from the limit cycle. 

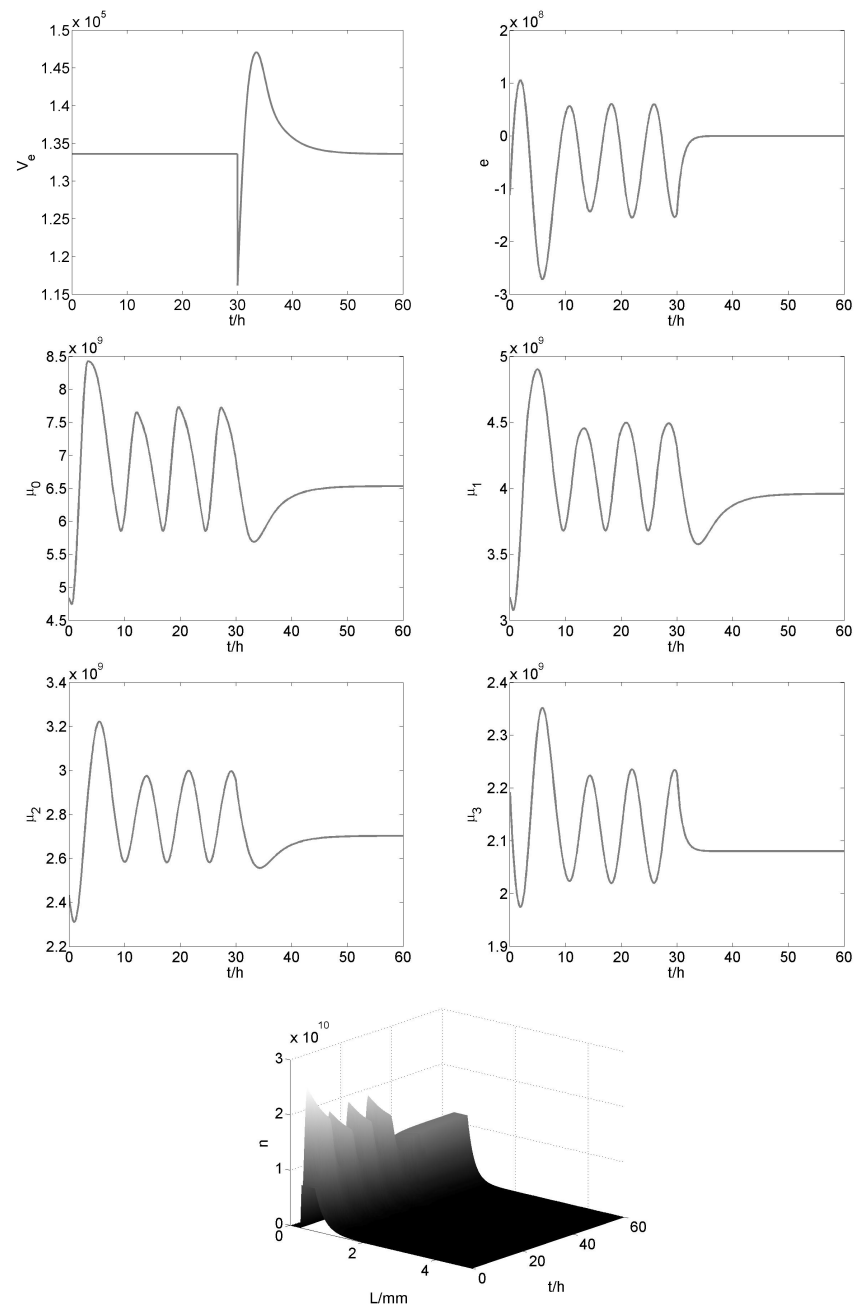

Fig. 8. Stabilization starting from the limit cycle

\section{CONCLUSION}

A new nonlinear control approach for continuous fluidized bed spray granulation with internal product classification has been developed and successfully tested. It uses a generalized distance measure, the discrepancy, and the associated stability theory, stability in the sense of Lyapunov with respect to two discrepancies, to design a nonlinear control law for the nonlinear distributed parameter system. As the proposed design guarantees only stability in the sense of Lyapunov with respect to the two chosen discrepancies, i.e. norms of the third moment of the particle size error distribution, conditions for pointwise convergences, i.e. convergence in a $L_{\infty}$-norm, have to be studied. It has been shown, that pointwise convergence can be achieved if and only if the zero dynamics associated with the chosen discrepancies are stable.

\section{REFERENCES}

[1] L. Mörl \& M. Mittelstrass \& J. Sachse, Zum Kugelwachstum bei der Wirbelschichttrocknung von Lösungen oder Suspensionen. Chem. Techn. 29, 1977, Heft 10, pp. 540-542.

[2] S. Heinrich \& M. Peglow \& M. Ihlow \& M. Henneberg \& L. Mörl, Analysis of the start-up process in continuous fluidized bed spray granulation by population balance modeling, Chem. Eng. Sci. 57, 2002, pp. 4369-4390.
[3] R. Radichkov \& T. Müller \& A. Kienle \& S. Heinrich \& M. Peglow \& L. Mörl, A numerical bifurcation analysis of continuous fluidized bed spray granulation with external product classification, Chem. Eng. Proc., Proc. 45, 2006, pp. 826-837.

[4] A.W. Vreman \& C.E. van Lare \& M.J. Hounslow, A basic population balance model for fluid bed spray granulation, Chem. Eng. Sci. 64, 2009, pp. 4389-4398.

[5] P. K. Pathath \& A. Kienle, A numerical bifurcation analysis of nonlinear oscillations in crystallization processes, Chem. Eng. Sci. 57, 2002, pp. 4391-4399.

[6] S. Palis \& A. Kienle, Stabilization of continuous fluidized bed spray granulation - a Lyapunov approach, NOLCOS - Bologna, 2010.

[7] S. Palis \& A. Kienle, Stabilization of continuous fluidized bed spray granulation, PBM - Berlin, 2010.

[8] S. Palis \& A. Kienle, Stabilization of continuous fluidized bed spray granulation with external product classification, Chem. Eng. Sci. 70, 2012, pp. 200-209.

[9] S. Palis \& A. Kienle, Diskrepanzbasierte Regelung der kontinuierlichen Kristallisation, at-Automatisierungstechnik 60 , 2012.

[10] S. Palis \& A. Kienle, $H_{\infty}$ loop shaping control for continuous fluidized bed spray granulation with internal product classification, Industrial \& Engineering Chemistry Research, 2011, under revision.

[11] R. Eek, Control and dynamic modeling of industrial suspension crystallizers, PhD thesis, TU Delft, 1995.

[12] P. Christofides \& N. El-Farra \& M. Li \& P. Mhaskar, Modelbased control of particulate processes, Chem. Eng. Sci. 63, 2008, pp. 1156-1172.

[13] U. Vollmer \& J. Raisch, Population balance modeling and $H_{\infty}$ controller design for a crystallization process, Chem. Eng. Sci. 57 , issue 20, Oct. 2002, pp. 4401-4414.

[14] A. D. Randolph \& M. A. Larson, Theory of particulate processes, New York: Academic Press, Inc., 1988.

[15] A. Balogh \& M. Krstic, Infinite-dimensional backstepping-style feedback transformations for a heat equation with an arbitrary level of instability, European Journal of Control 8, pp. 165-177, 2002.

[16] J. de Halleux \& C. Prieur \& J-M. Coron \& B. d'Andra-Novel \& G. Bastin, Boundary feedback control in networks of open channels, Automatica 39, pp. 1365-1376, 2003.

[17] A. Movchan, Stability of processes with respect to two metrics, Journal of Applied Mathematics and Mechanics 24, 1960, pp. 1506-1524.

[18] T. Sirasetdinov, Stability of systems with distributed parameters, Nauka Novosibirsk, 1987.

[19] A. Martynjuk \& R. Gutovski, Integral inequalities and stability of motion, Naukowa Dumka, 1979

[20] A. Isidori, Nonlinear Control Systems: An Introduction, Springer, 1995

[21] M. Krstic \& H. Deng, Stabilization of nonlinear uncertain systems, Springer, 1998

[22] E.D. Sontag, A universal construction of Artstein's theorem on nonlinear stabilization, Systems and Control Letters, 1989, 13, $117-123$. 